\title{
Metamaterial-inspired Electrically Small Canopy Antenna with Active Internal Element for Bandwidth Enhancement
}

\author{
Peng Jin and Richard W. Ziolkowski \\ Department of Electrical and Computer Engineering, The University of \\ Arizona, 1230 E. Speedway Blvd., Tucson, AZ, 85721-0104 \\ E-mail: pjin@ece.arizona.edu, ziolkowski@ece.arizona.edu
}

\section{Introduction}

Electrically small antennas (ESAs) are attractive in applications because of their compact size. On the other hand, because of their electrically small size, ESAs by themselves are generally poorly matched to a source. They are generally highly reactive, which means that is is hard to make them resonate (zero total reactance); and they have very low radiation resistance, which further exacerbates the matching problem, as well as prevents an efficient transduction of electrical power into radiated field power. Consequently, ESAs generally require an external matching network to ensure that they have a low return loss relative to their sources. A tremendous amount of effort has been reported in the literature on designing and using external matching networks to achieve ESA reactance compensation and resistance matching. Recently, several metamaterial-inspired ESAs were proposed [1], [2]. The ESA reactance compensation and resistance matching problems are addressed by introducing a reactive parasitic element in the very near field of the driven element. Because several of these designs rely on lumped elements, the latter and their integration into the parasitic element are refereed to as an internal matching network.

Although the metamaterial-inspired ESAs can be made radiate efficiently, their bandwidth performance remains limited by their electrical size and the Chu-based lower bound [3], [4]. This limit is now known to be: $Q_{c h u}=\eta_{\text {rad }}\left(1 / k a^{3}+1 / k a\right)$, where $k$ is the free space wavenumber, $a$ is the minimum radius of a sphere that completely encloses the antenna, and $\eta_{\text {rad }}$ is its radiation efficiency. The fractional $3 d B$ bandwidth for an ESA is limited by $F B W_{3 d B}=2 / Q_{c h u} \sim 2(k a)^{3} / \eta_{\text {rad }}$. Therefore, very small $k a$ ESAs automatically have very limited bandwidth even if their $Q$ values approach the limit $Q_{c h u}$. For example, for $k a=0.1$ and $\eta=1$, the bandwidth is limited to $0.2 \%$. This limit can be overcome by using Non-Forster matching networks as described, for example, in [5]. A metamaterial-inspired Z antenna with an active internal matching network was proposed in [6]. In this paper, the metamaterial-inspired, active internal matching network, canopy antenna introduced in [2] is discussed. The canopy antenna has $k a=0.0467$ and with the active internal matching network, has over a $10 \%$ fractional bandwidth.

\section{Canopy Antenna}

It was pointed out in [6] that the allowed error in the internal matching network design, $E \%$, must be limited by the desired antenna bandwidth as: $F B W_{10 d B} \geq E \%$. This error value would then maintain at least a $-10 d B$ return loss in the frequency 
band of interest. Since the fractional bandwidth is proportional to the inverse of the $Q$ value, it is desired to have the antenna's $Q$ value approach the $Q_{c h u}$ limit value. The Z antenna in [6] has a $Q_{\text {ratio }}=Q / Q_{c h u} \approx 11.2$. Thus, to account for the error in modeling, a $\mathrm{Z}$ antenna with $k a=0.267$ is needed to ensure enough bandwidth to account for this error. With a lower $Q$ ratio value, the antenna can endure a larger error in the modeling and, equivalently, in the fabrication, i.e., for a given error level, a large enough $k a$ value must be obtained.

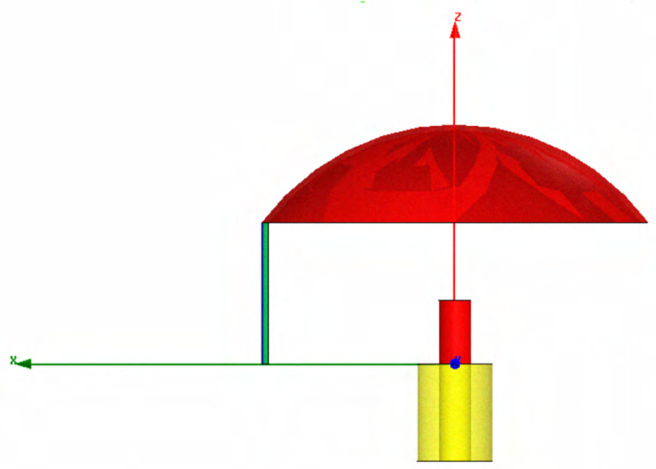

(a)

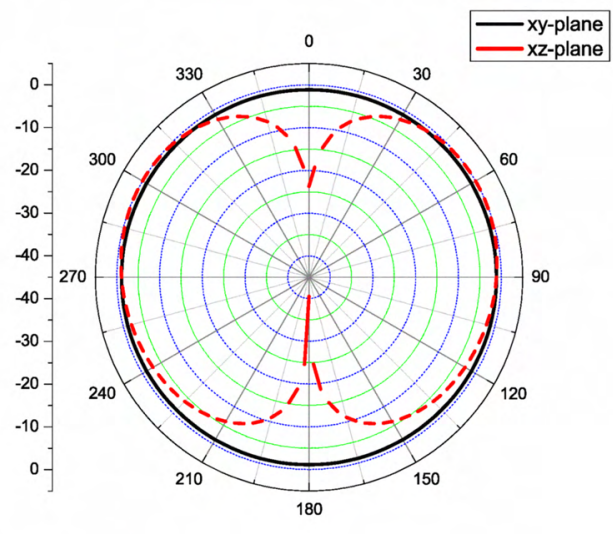

(b)

Figure 1: One-leg canopy antenna: (a) Side view of the one-leg canopy antenna, (b)Radiation patterns

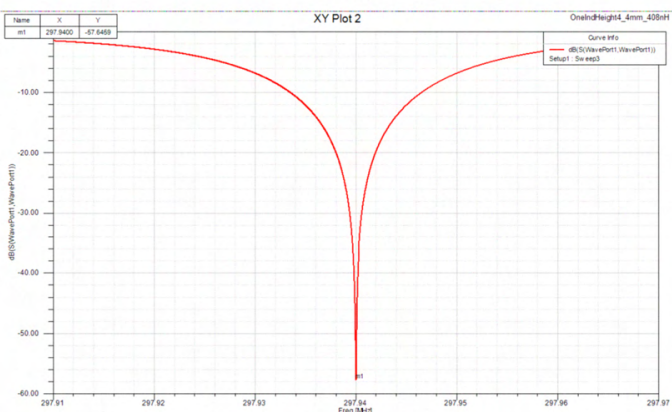

(a)

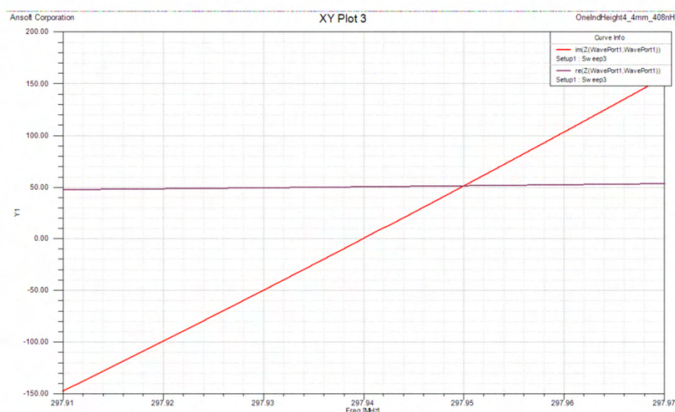

(b)

Figure 2: One-leg canopy antenna: (a) $S_{11}$ values, (b) complex input impedance values

The metamaterial-inspired $\mathrm{Z}$ antenna is composed of an electrically small coax-fed monopole and an inductive, resonant parasitic element located in the very near field of the monopole. Reactance compensation and resistance matching are achieved by the inductive parasitic element, i.e., the internal matching network. Based on this idea, the canopy antenna design shown in Fig. 1 (a) was obtained. The HFSS simulation results are shown, respectively, in Figs. 1(b) and 2. This canopy antenna resonates at $\approx 297 \mathrm{MHz}$ with $\eta_{\text {rad }} \approx 97 \%$ and has $k a=0.0467$. According to the $S_{11}$ and $Z_{i n}$ curves, it is well matched at its resonant frequency to the assumed $Z_{s}=50 \Omega$ source. Its far field pattern has an electrically small monopole pattern. The canopy antenna has a $Q_{\text {ratio }} \approx 1.75$. Compared with the $Q_{\text {radio }} \approx 11.2$ for the 
$\mathrm{Z}$ antenna, the improvement is significant and it sets the stage for achieving the active internal matching network over a very large bandwidth.

\section{Active Internal Matching Network}

Although the canopy antenna has $Q_{\text {ratio }} \approx 1.75$, its $10 \mathrm{~dB}$ bandwidth is only about $0.0045 \%$ because of its very small $k a$ value. Such a small bandwidth limits the usefulness of the canopy antenna for any real application. As pointed out in [6], if the lumped inductor $L$ is frequency dependent such that

$$
L \sim \xi\left(\frac{1}{f}\right)^{2}
$$

where $\xi$ is a constant over the desired frequency band, the canopy antenna can be nearly completely matched to the source and, hence, can have a high overall efficiency for those frequencies.

A set of HFSS simulations is performed to generate the required $L-f$ sweep. In reality, the frequency dependent relation (1) is never perfectly attained. We have found that instead of (1), the more precise curve fitting result

$$
L=\frac{\xi_{1}}{f^{2}}+\xi_{0}
$$

is obtained for the near field resonant parasitic elements. For instance, the $L-f$ sweep obtained in this manner for the $k a=0.0467$ one-leg canopy antenna is shown in Fig. 3(a). The constants $\xi_{1}=3.6702 \times 10^{7}$ and $\xi_{0}=-5.4525$. Moreover, we have found that because of numerical errors and tolerance errors in the inductance values of any purchased inductor, there will always be errors $(E \%)$ between the real inductor value and the inductor value determined by (2). Consequently, to ensure that $S_{11} \leq-10 d B$ at each frequency in the band of interest, the fractional bandwidth must satisfy: $F B W_{10 d B} \geq E \%$ [6]. The error in the resonance frequency, as calculated with the curve fit shown in Fig. 3(a), is shown in Fig. 3(b) along with the corresponding $F B W_{10 d B}$ values. One observes that the resonance frequency errors are substantially below the $F B W_{10 d B}$ values, thus ensuring that the one-leg canopy antenna would be resonantly well-matched to the source over more than a $10 \%$ bandwidth if a frequency dependent inductor with values given by (2) were introduced into it as an internal matching element. As shown [6], the necessary frequency dependent inductor defined by (2) can be achieved with a series connected negative capacitor and negative inductor which can be realized with the negative impedance inverter (NIC) element introduced in [7]. This NIC element would be placed beneath the ground plane and would be connected to the canopy through a copper leg (the inductor would be replaced by the copper leg above the ground plane); and yet it is small enough to fit within the minimum-enclosing sphere so as not to increase the overall electrical size of the antenna system. We have run a variety of HFSS simulations with the canopy leg being partly copper and partly inductor to verify this potential realization. 


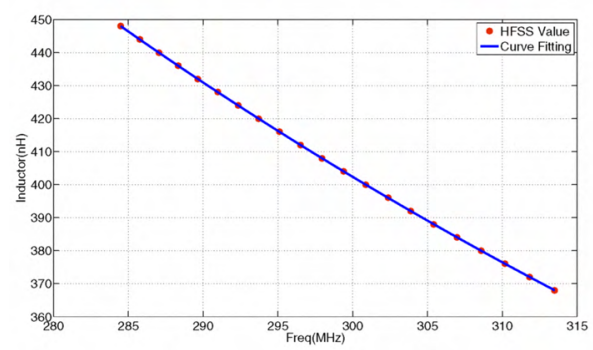

(a)

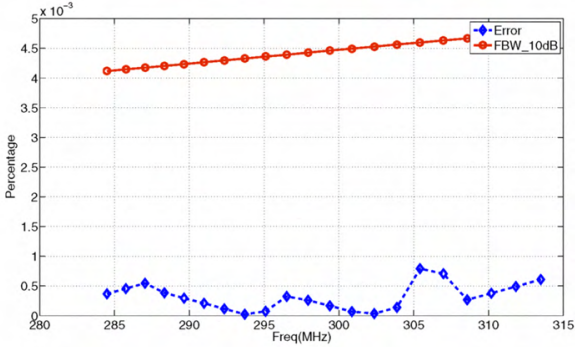

(b)

Figure 3: One-leg canopy antenna with $k a=0.0467$ : (a) Inductor-frequency (L-F) sweep, (b) Comparison of the curve fitting errors and the $F B W_{10 d B}$ values

\section{Conclusion}

In this paper, a very electrically small canopy antenna is introduced. The canopy antenna radiates at $\approx 297 M h z$ with a $Q_{\text {ratio }} \approx 1.75$. An active internal matching network is applied to this canopy antenna; over a $10 \%$ fractional bandwidth is obtained for this $\operatorname{ESA}(a \sim \lambda / 137)$.

\section{References}

[1] A. Erentok and R. W. Ziolkowski, "Metamaterial-inspired efficient electricallysmall antennas," IEEE Trans. Antennas Propag., pp. 691-707, Mar 2008.

[2] P. Jin and R. W. Ziolkowski, "Low-q, electrically small, efficient near-field resonant parasitic antennas," submitted to IEEE Trans. Antennas Propag., December 2008 .

[3] L. J. Chu, "Physical limitations of omni-directional antennas," J. Appl. Phys., pp. 1163-1175, 1948.

[4] H. A. Wheeler, "Fundamental limitations of small antennas," Proc. IRE, pp. 1479-1484, 1947.

[5] J. T. Aberle and R. Lopesinger-Romak, Antenna with Non-Foster Matching Networks. San Rafael, CA: Morgan \& Claypool Publishers, 2007.

[6] P. Jin and R. W. Ziolkowski, "Broadband, efficient, electrically small metamaterial-inspired antennas facilitated by active near-field resonant parasitic elements," in preparation, 2008.

[7] A. Larky, "Negative-impedance converters," IRE Trans. Circuit Theory, pp. 124-131, Sep. 1957. 